\title{
Manejo de la pancreatitis aguda litiásica en la embarazada
}

\author{
Camila Fernández ${ }^{1,2}$, Jorge Carvajal ${ }^{2}$, Dr. Fernando Crovari ${ }^{4}$, Claudio Vera ${ }^{2,3}$, José Poblete ${ }^{2}$.
}

Resumen: La pancreatitis aguda (PA) es una patología poco frecuente durante el embarazo, siendo la colelitiasis una de sus principales etiologías y Chile uno de los países con más altas prevalencias de colelitiasis en el mundo. Esta patología clásicamente ha reportado altas tasas de mortalidad materno-fetal, así como también riesgo de morbilidad fetal. Sin embargo, gracias a los avances en terapia de soporte y mejoras en unidades de neonatología, en la actualidad el pronóstico con un adecuado tratamiento es alentador. Nos parece imperativo tener protocolos adecuados de toma de decisión en la embarazada con pancreatitis aguda, puesto que, a pesar de ser una patología de baja prevalencia, un correcto enfrentamiento puede mejorar el resultado materno-fetal. El presente trabajo propone una guía de manejo clínico interdisciplinario de la embarazada con pancreatitis y aporta una revisión actual sobre este tema.

Palabras clave: pancreatitis aguda; el embarazo; pancreatitis en el embarazo.

Abstract: Acute pancreatitis (PA) is a rare disease during pregnancy, with cholelithiasis being one of its major etiologies and Chile one of the countries with the highest prevalence of cholelithiasis in the world. This pathology has traditionally reported high rates of maternalfetal mortality, as well as the risk of fetal morbidity. However, thanks to the advances in supportive therapy and improvements in neonatal units, the prognosis with an adequate treatment is now encouraging. It seems imperative to have adequate decision-making protocols in the pregnant woman with acute pancreatitis, since despite being a pathology of low prevalence a correct confrontation can improve the maternal-fetal outcome. This paper proposes an interdisciplinary clinical management guide for pregnant women with pancreatitis and provides a current review on this topic.

Keywords: Acute pancreatitis; Pregnancy; Pancreatitis in pregnancy.

Fecha de envío: 4 de abril de 2017 - Fecha de aceptación: 31 de agosto de 2017

\section{Introducción}

La pancreatitis aguda (PA) es una patología caracterizada por grados diversos de inflamación aguda del tejido pancreático y peri-pancreático; que, en sus formas graves, puede llevar a un importante compromiso sistémico (Sarr, 2013). En población general su incidencia se estima en 13-45 por 100.000, siendo mayor en poblaciones con alta incidencia de colelitiasis, como lo es la chilena (Yadav \& Lowenfels, 2013).

Durante el embarazo, la incidencia de pancreatitis es variable con tasas descritas entre 25 a 100 casos por 100.000 embarazadas, siendo más frecuente a mayor edad gestacional (Ramin et al., 1995; Hernández et al., 2007; Turhan et al., 2010). En Chile no existen datos de incidencia, sin embargo, es reconocido que es uno de los países con más altas tasas de colelitiasis en el mundo, especialmente en el grupo de mujeres en edad fértil, con tasas de hasta el 30-44\%, las cuales son hasta 10 veces mayores durante el embarazo y puerperio (Valdivieso et al., 1993; Ramin \& Ramsey, 2001; Carbonell et al., 2012). En este contexto parece lógico suponer que nuestras tasas de pancreatitis en el embarazo son mayores que las reportadas en otras poblaciones.

La PA durante el embarazo tiene un amplio espectro clínico, ha sido reportada como causa de muerte materna y fetal, con una letalidad que oscila entre el $37 \%$ y $11-50 \%$ respectivamente (Scott, 1992; Ramin et al., 1995; Eddy et al., 2008). En la actualidad, gracias al diagnóstico precoz, adecuado soporte materno y mejores

$(1,2,3)$ Programa de Medicina Materno Fetal, Unidad de Medicina Materno Fetal, Programa de Salud Basada en Evidencia. División de Obstetricia y Ginecología, Facultad de Medicina. Pontificia Universidad Católica de Chile.

(4) Departamento de Cirugía Digestiva, División de Cirugía, Facultad de Medicina. Pontificia Universidad Católica de Chile. Autor de Correspondencia: cfernandeznikli@gmail.com 
unidades de neonatología se ha logrado reducir significativamente la letalidad de esta asociación, con cifras de $1 \%$ y $0-23 \%$ respectivamente (Scott, 1992; Ramin et al., 1995; Eddy et al., 2008) (Tabla 1). Lamentablemente, la mortalidad no es el único riesgo

Tabla 1. Pancreatitis aguda durante el embarazo, reportes de casos. asociado a esta patología y a pesar de la importante mejora que hemos logrado, debemos tener en cuenta los riesgos de prematurez, óbito fetal y otras morbilidades, como se resume en la Tabla 2.

\begin{tabular}{|c|c|c|c|c|c|c|c|c|c|c|c|c|}
\hline & \multirow[b]{2}{*}{$\mathrm{N}$} & \multirow[b]{2}{*}{ Años } & \multicolumn{4}{|c|}{ Etiología (\%) } & \multicolumn{3}{|c|}{ Trimestre (\%) } & \multirow[b]{2}{*}{ 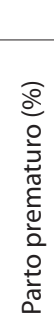 } & \multirow[b]{2}{*}{ 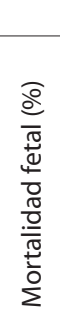 } & \multirow[b]{2}{*}{ 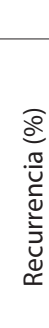 } \\
\hline & & & 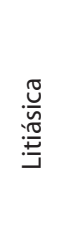 & $\begin{array}{l}\frac{0}{0} \\
\frac{O}{0} \\
\frac{O}{0} \\
\frac{U}{\alpha}\end{array}$ & 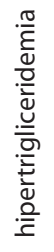 & $\begin{array}{l}\stackrel{0}{0} \\
\stackrel{0}{0} \\
\stackrel{0}{0} \\
\stackrel{0}{0}\end{array}$ & - & $=$ & $\equiv$ & & & \\
\hline Eddy et al., 2008 & 101 & $1992-2001$ & 66 & 12 & 4 & 17 & 24 & 18 & 43 & 18 & 4,7 & 26 \\
\hline Ramin et al.,1995 & 42 & 1983-1993 & 67 & 7 & 0 & 17 & 19 & 26 & 55 & 14 & 9,5 & NR \\
\hline Igbinosa et al., 2013 & 29 & 2005-2009 & 70 & 3,4 & 0 & 24 & 13 & 3 & 48 & 3 & 0 & 6 \\
\hline Qihui et al.,2012 & 26 & 1997-2009 & 77 & 0 & 11 & 12 & 0 & 29 & 0 & 29 & 23 & NR \\
\hline Hernández et al., 2007 & 21 & 1992-2001 & 57 & 4,7 & 0 & 28 & 6 & 19 & 38 & 19 & 4,7 & 50 \\
\hline
\end{tabular}

NR: no reportada

Tabla 2. Complicaciones materno-fetales asociadas a pancreatitis aguda en la embarazada.

\begin{tabular}{|l|l|}
\hline Maternas & Fetales \\
\hline - Muerte & \\
- Sepsis & Óbito Fetal \\
- Colangitis & Parto prematuro \\
- SRIS & \\
- Falla orgánica múltiple & \\
- Complicaciones locales & \\
\hline
\end{tabular}

Se requiere de alta sospecha de esta condición, y de protocolos adecuados de manejo de la embarazada con pancreatitis aguda, para mejorar el resultado materno y fetal.

El presente trabajo tiene como objetivo proponer una guía de manejo clínico interdisciplinario de la embarazada con pancreatitis, sobre la base de la mejor evidencia disponible publicada en la literatura médica.

\section{Etiopatogenia de la pancreatitis aguda en el embarazo}

Las etiologías de la pancreatitis aguda en el embarazo son similares a las reportadas en población general (Ramin et al., 1995;
Ramin \& Ramsey, 2001; Hernández et al., 2007; Eddy et al., 2008; Igbinosa, et al., 2013). Se estima que más del $70 \%$ de ellas son secundarias a litiasis biliar; reportes recientes que incluyen más de 220 pacientes confirman que el $57-77 \%$ de los casos de pancreatitis aguda durante el embarazo se deben a litiasis biliar. Le siguen en frecuencia las pancreatitis idiopáticas (12-28\%), por abuso de alcohol (0-12\%) y secundarias a hipertrigliceridemia (4\%) (Tabla 1). Existen otras causas de pancreatitis aguda durante el embarazo, reportadas con mucho menor frecuencia como el hiperparatiroidismo, trauma, fármacos, infecciones, etc.(Ramin et al., 1995; Hernández et al., 2007; Eddy et al., 2008; Qihui et al., 2012).

Es bien conocido que el embarazo representa un estado litogénico, el que se explica por cambios fisiológicos propios de este (Valdivieso et al., 1993; Papadakis et al., 2011).

Durante la gestación, aumentan los niveles de progesterona, hormona que produce relajación del musculo liso, afectando la pared vesicular; la progesterona además inhibe parcialmente la colecistoquinina, la cual tiene la función de regular la contractilidad vesicular. Los dos mecanismos mencionados determinan una vesícula hipotónica, aumentada de tamaño y con una evacuación enlentecida e incompleta, lo cual favorece la estasis biliar. Por otra parte, se ha reportado aumento de la secreción hepática 
de colesterol comparada con la de ácidos biliares, llevando a saturación biliar. Finalmente, hay mayor formación de cristales de colesterol, los cuales formarán los cálculos biliares. Estos cálculos, especialmente los micro-cálculos, así como el barro biliar, pueden obstruir el conducto pancreático desencadenando la inflamación y liberación de enzimas pancreáticas, propias de la pancreatitis aguda (Ramin et al., 1995; Vonlaufen, et al., 2008).

\section{Consideraciones diagnósticas en el embarazo}

El diagnóstico de pancreatitis aguda se basa en la presencia de dos de tres criterios diagnósticos: cuadro clínico, exámenes de laboratorio y/o estudio de imágenes (Working Group IAP/APA, 2013; Sarr, 2013).

La clínica es generalmente poco específica, se presenta con dolor en hemiabdomen superior y/o epigastrio, de carácter mal definido, no cólico, de inicio progresivo hasta máxima intensidad, pudiendo ser mantenido por días. Puede existir irradiación en cinturón a espalda en el 50\% de los casos (Ramin et al., 1995; Hernández et al., 2007). El dolor es agravado por la ingesta de líquido o alimentos y por la posición supina. Al dolor suelen asociarse náuseas y vómitos, anorexia, distención abdominal e incluso fiebre en el 40-50\% de las pacientes (Ramin et al., 1995; Papadakis et al., 2011; Working Group IAP/APA, 2013).

Frente a un cuadro clínico sospechoso, se deben solicitar exámenes de laboratorio; una elevación de amilasa y/o lipasa mayor o igual a 3 veces sobre su valor normal confirma el diagnóstico (Ramin et al., 1995; Hernández et al., 2007; Papadakis et al., 2011;Working Group IAP/APA, 2013; Sarr, 2013). La lipasa tiene la ventaja de ser más específica y de permanecer elevada por más tiempo que la amilasa (Working Group IAP/APA, 2013; Sarr, 2013). Se ha reportado que en embarazos normales los niveles de estas enzimas permanecen normales o muy levemente elevados (Karsenti et al., 2001; Larsson et al., 2008).

Dentro del estudio de laboratorio se deben incluir, entre otros, hemograma, proteína $C$ reactiva (PCR), función hepática, calcemia y perfil lipídico.

El estudio de imágenes se debe limitar inicialmente a la ecografía abdominal, por ser un examen inocuo, de bajo costo y alta disponibilidad, que nos permite por una parte, descartar otras causas de dolor abdominal, y por otra, evaluar la presencia de litiasis biliar, considerando que esta es la etiología más frecuente. En caso de duda diagnóstica, se puede recurrir a la tomografía axial computada, la cual tiene la ventaja de evaluar adecuadamente el páncreas, su desventaja radica en el costo y en la radiación emitida.
Ante la confirmación de una pancreatitis aguda, se debe dilucidar la etiología, mediante anamnesis, exámenes de laboratorio e imágenes. En la anamnesis es importante preguntar por cuadros anteriores de pancreatitis aguda, colelitiasis conocida, consumo de alcohol, fármacos o drogas, antecedentes de trauma abdominal reciente, entre otros. Los exámenes de laboratorio permitirán estudiar la presencia de una hipertrigliceridemia y la ecografía permitirá evaluar la presencia o signos sugerentes de litiasis de la vía biliar.

\section{Estudio de imágenes}

Ecografía abdominal: representa el examen de primera línea para el estudio de la embarazada con pancreatitis aguda; la ecografía no usa radiación ionizante, es de bajo costo, no invasivo y de alta disponibilidad. Además, tiene muy buena sensibilidad (90-100\%) en la detección de colelitiasis ( $>3 \mathrm{~mm}$ ) y dilatación de vía biliar, la cual se mantiene en mujeres embarazadas (Pitchumoni \& Yegneswaran, 2009; Koo et al., 2010). Su desventaja es que se logra visualizar el páncreas solo en $30-40 \%$ de los casos y tiene baja sensibilidad (< 60\%) para detectar el barro biliar y la coledocolitiasis (Balthazar et al., 1990; Koo et al., 2010; Working Group IAP/APA, 2013). El principal objetivo de la ecografía será evaluar la etiología litiásica, más que confirmar la pancreatitis.

Tomografía axial computada (TAC): es un examen no invasivo, muy útil para evaluar el páncreas, permitiendo el diagnóstico de inflamación, colecciones y necrosis pancreática. LaTAC es útil no solo para el diagnóstico, sino también para el pronóstico de la pancreatitis aguda. Balthazar et al.(1990) describió un índice de gravedad para la pancreatitis aguda, en el cual, según las características morfológicas del páncreas (clasificación de Balthazar) y el porcentaje de necrosis (Tabla 3), se otorga un puntaje que se relaciona con el pronóstico de morbilidad y mortalidad (Tabla 4). (Balthazar et al., 1990). Las desventajas de la TAC son que requiere contraste endovenoso para el diagnóstico de necrosis, usa irradiación ionizante, tiene mayor costo y subestima la extensión de necrosis y la gravedad.

En cuanto al contraste yodado endovenoso en el embarazo, se sabe que atraviesa la placenta y es considerado por la FDA como un medicamento categoría B. Existiría un riesgo teórico de depresión en la función tiroidea en fetos expuestos, el cual no se ha logrado demostrar. (Lazarus et al., 2007; Uribe et al., 2009).

En cuanto a la irradiación, una TAC de abdomen y pelvis en promedio produce una exposición fetal a 0,8 rads, lo cual está lejos de la cantidad máxima permitida, que es 5 rads (Lazarus et al., 2007; Uribe et al., 2009). Sin embargo, algunos estudios han sugerido que la exposición fetal a la radiación ionizante podría ser muy variable, incluso llegando a los 4,2 rads (Lazarus et al., 2007). 
Tabla 3. Índice de gravedad de la pancreatitis aguda según hallazgos del TAC.

\begin{tabular}{|c|c|c|}
\hline \multicolumn{3}{|c|}{ Clasificación de Balthazar } \\
\hline Grado & Descripción del páncreas & Puntos \\
\hline A & Páncreas normal & 0 \\
\hline B & $\begin{array}{l}\text { Aumento de tamaño focal o difuso } \\
\text { del páncreas. Sin evidencia de } \\
\text { enfermedad peri-pancreática }\end{array}$ & 1 \\
\hline $\mathrm{C}$ & $\begin{array}{l}\text { Alteraciones pancreáticas intrínsecas } \\
\text { asociadas con: aumento de la } \\
\text { densidad peri pancreática difusa } \\
\text { y parcial, que representa cambios } \\
\text { inflamatorios en la grasa }\end{array}$ & 2 \\
\hline D & Colección líquida única mal definida. & 3 \\
\hline $\mathrm{E}$ & $\begin{array}{l}\text { Dos o múltiples colecciones líquidas } \\
\text { pobremente definidas o presencia de } \\
\text { gas en o adyacente al páncreas. }\end{array}$ & 4 \\
\hline \multicolumn{3}{|c|}{ Necrosis al TAC } \\
\hline & $\%$ & Puntos \\
\hline & 0 & 0 \\
\hline & $<30$ & 2 \\
\hline & $30-50$ & 4 \\
\hline & $>50$ & 6 \\
\hline
\end{tabular}

Tabla 4. Morbilidad y mortalidad según índice de severidad

\begin{tabular}{|l|l|l|l|}
\hline Grado & Puntos & Morbilidad & Mortalidad \\
\hline Muy bajo & $0-1$ & $0 \%$ & $0 \%$ \\
\hline Bajo & $2-3$ & $8 \%$ & $3 \%$ \\
\hline Medio & $4-6$ & $35 \%$ & $6 \%$ \\
\hline Alto & $7-10$ & $92 \%$ & $17 \%$ \\
\hline
\end{tabular}

El índice de severidad considera el puntaje obtenido en: 1) la clasificación de Balthazar que otorga puntaje de 0 a 4 según el aspecto del páncreas en el TAC sin contraste y 2 ) el porcentaje de necrosis del páncreas ( 0 a 6 puntos), evaluado en TAC con contraste (ver Tabla 3). El puntaje obtenido determina la severidad de la pancreatitis y tiene muy buena correlación con morbilidad y mortalidad.

Es por esto que sugerimos no realizar de rutina la TAC en la embarazada con pancreatitis, sino que reservarla para casos de pancreatitis grave posterior a las $72 \mathrm{~h}$ de evolución; $\mathrm{APACHE} \geq 8$, Ranson $>3$, falla orgánica, o si presenta deterioro clínico significativo (Working Group IAP/APA, 2013).

Resonancia magnética (RM): es un examen no invasivo, no requiere contraste endovenoso en caso de colangio-resonancia, no usa radiación ionizante y logra una excelente visualización del páncreas. Sin embargo, es de alto costo, menor accesibilidad y mayor tiempo de realización, además aún no está estandarizada en evaluación pronóstica. La principal utilidad de este examen es ante la sospecha de coledocolitiasis en la forma de colangio-resonancia. Respecto a la RM en el embarazo, no existe evidencia de que la exposición a campos magnéticos tenga algún efecto dañino para el feto; siendo la principal preocupación el aumento de temperatura que los pulsos de radiofrecuencia podrían producir en tejidos. Es por esta razón que se sugiere evitar su uso antes de las 18 semanas de gestación (Garel et al., 1998; Uribe et al., 2009).

\section{Manejo}

Una vez realizado el diagnóstico de pancreatitis aguda e identificada la causa, se debe clasificar a la mujer según la gravedad del cuadro, lo cual determina el tratamiento a seguir. Se han creado múltiples criterios para determinar gravedad, siendo en la actualidad los más utilizados el Score APACHE II, Ranson y la identificación de falla orgánica o de complicaciones locales o sistémicas que permiten clasificar la pancreatitis en leve, moderadamente severa o severa según el consenso ATLANTA 2012 (Sarr, 2013)(Tabla 5). Debido a que durante el embarazo varios de los parámetros vitales así como los de laboratorio están alterados, sugerimos usar como criterio de gravedad la aparición de falla multiorgánica, la que puede definirse usando los criterios sugeridos por la OMS para la identificación de Near miss (World Health Organization, 2011). En caso de presentar cualquiera de estos criterios, se clasifica como una pancreatitis aguda grave, que requiere manejo en una Unidad de Paciente Crítico. A continuación, indicamos los principales elementos a considerar en el manejo de la pancreatitis aguda durante el embarazo (Tabla 6).

Tabla 5. Criterios gravedad PA

\begin{tabular}{|c|c|}
\hline APACHE II & $\geq 8$ \\
\hline Ranson Score & $\geq 3$ \\
\hline Score Marshall & $\geq 2$ (Define falla orgánica Atlanta 2012) \\
\hline \multicolumn{2}{|c|}{ Clasificación ATLANTA 2012} \\
\hline a. Leve & $\begin{array}{l}\text { Ausencia falla orgánica y de } \\
\text { complicaciones locales o sistémicas }\end{array}$ \\
\hline $\begin{array}{l}\text { b. Moderadamente } \\
\text { Severa }\end{array}$ & $\begin{array}{l}\text { Falla orgánica transitoria (<48h) o } \\
\text { complicación local o sistémica en } \\
\text { ausencia de falla orgánica persistente. }\end{array}$ \\
\hline c. Severa & Falla orgánica persistente ( $>48$ hr) \\
\hline \multicolumn{2}{|c|}{ Falla orgánica - Near miss OMS } \\
\hline Shock & $\begin{array}{l}\text { Hipoperfusión severa } \\
\text { Lactato }>45 \mathrm{mg} / \mathrm{dl} \\
\text { Acidosis severa }(<7,1)\end{array}$ \\
\hline Insuficiencia Pulmonar & $\begin{array}{l}\text { Saturación } \mathrm{O}_{2}<90 \% \text { x } 60 \mathrm{~min} \\
\mathrm{PAO}_{2} / \mathrm{FiO}_{2}<200 \mathrm{~mm} \mathrm{Hg}\end{array}$ \\
\hline Falla Renal & Creatininemia $>3,5 \mathrm{mg} / \mathrm{dl}$ \\
\hline \multicolumn{2}{|l|}{ Complicación sistémica } \\
\hline CID & $\begin{array}{l}\text { Plaquetas }<50.000 \text {, Fibrinógeno }<1 \mathrm{~g} / \mathrm{Lt} \text {, } \\
\text { PDF }>80 \mu \mathrm{g} / \mathrm{mL}\end{array}$ \\
\hline Alteración metabólica & Calcemia $<7,5 \mathrm{mg} / \mathrm{dl}$ \\
\hline
\end{tabular}

$\mathrm{PAO}_{2}$ : Presión arterial oxígeno, $\mathrm{FiO}_{2}$ : Fracción inspirada de oxigeno.

APACHE Il: Acute Physiology And Cronic Health Evaluation: Sistema que permite cuantificar la gravedad de la enfermedad a través de 12 variables fisiológicas que expresan la intensidad de la enfermedad; 6 examen clínico +6 exámenes de laboratorio, a esto se suma puntaje por edad y presencia de enfermedades crónicas concomitantes. Ventaja permite evaluación en primeras $24 \mathrm{hr}$. Ranson Score: Basado en la medición de 11 factores; 5 controlados en la admisión y 6 chequeados a las 48 hr, por esto tiene la desventaja de requerir 48 hr de observación.

Marshall: Incluye evaluación sistemas renal (creatininemia), respiratorio (PAFi) y cardiovascular (PAs) y requerimiento de oxigeno.

Un Score Ranson $>3$ o un APACHE II > 8, se asocia a mortalidad mayor al $15 \%$ (Ranson et al., 1974) 
Tabla 6. Esquema de manejo de la pancreatitis aguda durante el embarazo

\begin{tabular}{|c|c|c|}
\hline Procedimiento & Descripción & Elementos principales \\
\hline $\begin{array}{l}\text { Reposición de } \\
\text { fluidos }\end{array}$ & Suero ringer lactato & $\begin{array}{l}5-10 \mathrm{cc} / \mathrm{kg} / \mathrm{hr} \text { primeras } \\
4 \mathrm{hr} \\
80 \mathrm{cc} / \mathrm{hr} \text { siguientes } 20 \mathrm{hr} \\
\text { Aporte total } 24 \mathrm{hr}: 2,5-4 \mathrm{Lt} \text {. }\end{array}$ \\
\hline Nutrición & $\begin{array}{l}\text { Preferir enteral } \\
\text { En caso de requerir } \\
\text { parenteral preferir } \\
\text { catéter periférico }\end{array}$ & $\begin{array}{l}\text { Inicio precoz. } \\
\text { Considerar que el tiempo } \\
\text { necesario para lograr } \\
\text { el aporte nutricional } \\
\text { necesario es } 3 \text { a } 4 \text { días }\end{array}$ \\
\hline Antibióticos & $\begin{array}{l}\text { Ceftriaxona } 1 \text { gr cada } \\
12 \mathrm{hr} \text { ev } \\
\text { Metronidazol } 500 \mathrm{mg} \\
\text { cada } 8 \mathrm{hr} \mathrm{ev}\end{array}$ & $\begin{array}{l}\text { Utilizar en caso de } \\
\text { pancreatitis grave y/o } \\
\text { asociadas a colangitis }\end{array}$ \\
\hline Analgesia & $\begin{array}{l}\text { Suero fisiológico } 250 \\
\mathrm{cc}+\text { Tramadol } 200- \\
300 \mathrm{mg} \text { a } 10 \mathrm{cc} / \mathrm{hr} \\
\text { + Odanex } 4 \mathrm{mg} \text { cada } \\
8 \mathrm{hr} \text { ev } \\
\text { + Paracetamol } 1 \mathrm{gr} \\
\text { cada } 8 \mathrm{hr} \mathrm{ev} \\
\text { + Morfina } 3 \mathrm{mg} \text { ev SOS }\end{array}$ & $\begin{array}{l}\text { En caso de mala respuesta } \\
\text { evaluar con equipo de } \\
\text { dolor. } \\
\text { Evaluar inicio PCA } \\
\text { morfina. }\end{array}$ \\
\hline $\begin{array}{l}\text { Resolución } \\
\text { quirúrgica }\end{array}$ & $\begin{array}{l}\text { En etiología litiásica } \\
\text { Colecistectomía } \\
\text { de preferencia vía } \\
\text { laparoscópica }\end{array}$ & $\begin{array}{l}\text { I trimestre: Diferir cirugía } \\
\text { a II trimestre. } \\
\text { II Trimestre: Resolución } \\
\text { previo al alta. } \\
\text { III Trimestre: Decisión } \\
\text { individualizada. }\end{array}$ \\
\hline
\end{tabular}

1. Reposición de fluidos: la reposición de volumen representa uno de los pilares fundamentales del tratamiento de las mujeres embarazadas con pancreatitis aguda, puesto que disminuye el riesgo de SIRS, falla orgánica y mortalidad. La reposición de volumen debe ser precoz y enérgica (Gardner, et al., 2008). Se sugiere iniciar reposición con cristaloides, de preferencia Ringer lactato, puesto estudios sugieren una reducción en la incidencia de SIRS al compararlo con suero fisiológico (Mutter, et al, 2013). Por otra parte, se recomienda evitar el uso de Hidroxialmidon (Voluven), dado que aumentaría el riesgo de falla renal y mortalidad en pacientes con sepsis en cuidados intensivos (Wu et al., 2011).

El volumen a usar en la reposición inicial de fluidos debe ser entre $5-10 \mathrm{cc} / \mathrm{Kg} / \mathrm{h}$ en las primeras $4 \mathrm{~h}$ ), luego se debe reducir el aporte, siendo en la mayoría de los pacientes la infusión total de 2500 a 4000 cc en 24 h suficiente para la resucitación inicial (Working Group IAP/APA, 2013).

2. Nutrición: se debe mantener a la paciente en ayuno e iniciar precozmente $(<48 \mathrm{~h}$ ) el apoyo nutricional. Las pacientes con pancreatitis aguda muestran un patrón de catabolismo de proteína elevado, un metabolismo de glucosa alterado y frecuentemente una digestión y absorción de nutrientes menor, todo lo anterior puede llevar a desarrollar déficit nutricional, siendo este aún más importante en pacientes embarazadas (Rinninella, et al., 2017).

Preferir la nutrición enteral sobre la parenteral, puesto que esta es más fisiológica, mantiene la flora intestinal habitual, la inmunidad e integridad de la mucosa intestinal y reduce la translocación bacteriana, así como los niveles de endotoxinas plasmáticas (AlOmran, et al., 2010; Working Group IAP/APA, 2013) Rinninella et al., 2017). Se ha observado tanto en modelos animales como en estudios prospectivos en humanos que durante un episodio de pancreatitis aguda la secreción de enzimas exocrinas desde el páncreas se ve suprimida, lo cual explica en parte la seguridad de la nutrición enteral. La nutrición enteral versus la parenteral reduce significativamente mortalidad 0.50 (95\% Cl 0.28- 0.91), falla orgánica múltiple 0.55 ( $95 \%$ Cl 0.37- 0.81), infección sistémica 0.39 (95\% Cl 0.23- 0.65), complicaciones sépticas locales 0.74 (95\% Cl 0.40-1.35) y días de hospitalización promedio en 2.37 días $(95 \% \mathrm{Cl}$ -7.18- 2.44) (Wu et al., 2011). Al decidir el inicio de nutrición enteral, se debe considerar que el tiempo necesario para lograr el aporte nutricional necesario es de 3-4 días. En cuanto a la ubicación del tubo de nutrición enteral, se ha observado en estudios clínicos randomizados y en un posterior meta-análisis que la sonda nasogástrica versus la nasoyeyunal no presenta diferencias significativas en mortalidad, tasa de aspiración traqueal, diarrea, exacerbación de dolor abdominal ni balance energético. Dado que la instalación de la sonda nasogástrica es técnicamente más fácil y no requiere procedimientos radiológicos o endoscópicos adicionales, en la actualidad esta es una alternativa válida. (Rinninella et al. 2017)

Por otra parte, un catéter venoso central (CVC) tiene mayor riesgo en la mujer embarazada comparada con la mujer no embarazada, tanto de trombosis, como de infección (Russo et al., 1999). Así, en mujeres embarazadas que requieran nutrición parenteral, se debe preferir el uso de catéter periférico, lo que se asociará a menor tasa de complicaciones comparado con el CVC ( $9 \%$ vs. $50 \%$ respectivamente) (Russo et al., 1999).

Se puede iniciar la realimentación oral en las embarazadas con pancreatitis cuando desaparezca el dolor abdominal, las náuseas y los vómitos, el paciente recupere el apetito y los marcadores inflamatorios y enzimas pancreáticas comiencen a mejorar, siendo lo más importante la mejoría clínica por sobre el laboratorio.

3. Antibióticos: la antibioterapia debe reservarse para los casos de pancreatitis grave y/o asociada a colangitis. Sugerimos como esquema de primera línea el uso de cefalosporinas de tercera generación en asociación con antianaerobio, Ceftriaxona 1 a 2 g/día y Metronidazol $500 \mathrm{mg} \mathrm{c} / 8 \mathrm{~h}$, puesto que son antibióticos seguros 
en el embarazo y cubren un amplio espectro de microorganismos (Burtin, et al., 1995; Vallano \& Arnau, 2009). Evitar el uso de aminoglucósidos, ampicilina y cefalosporinas de primera generación por su baja penetración al páncreas. Se desaconseja el uso de quinolonas durante el embarazo debido al riesgo de erosión de cartílagos y artropatía fetal (Vallano \& Arnau, 2009).

4. Analgesia: el control del dolor es un pilar fundamental del tratamiento en embarazadas con pancreatitis aguda. A pesar de que los opioides se evitaron por mucho tiempo en el tratamiento de la pancreatitis aguda por su posible efecto en la contracción del esfínter de Oddi, actualmente representan la primera línea de manejo en la embarazada. Una revisión sistemática reciente observó que en pacientes con pancreatitis aguda los opioides, comparados con otros analgésicos, reducen la necesidad de analgesia suplementaria, sin diferencia en el riesgo de complicaciones o efectos adversos serios (Basurto et al., 2013).

Sugerimos iniciar la analgesia en la embarazada con una infusión continua de Tramadol asociado a un antiemético y paracetamol horario, con morfina endovenosa de rescate. En caso de presentar mala respuesta, se puede utilizar una bomba de infusión analgésica controlada por la paciente (PCA) de morfina endovenosa (Tabla 6).

5. Resolución quirúrgica: existe controversia en cuanto a las indicaciones y mejor momento de las intervenciones quirúrgicas en la evolución de la pancreatitis aguda litiásica en la embarazada. En población general, se ha demostrado el beneficio de realizar la colecistectomía previo al alta, dado que el riesgo de recurrencia en las 6 primeras semanas alcanza el $30 \%$, a lo cual se suma un riesgo de colecistitis aguda de 10-25\% (van Baal et al., 2012).

Es importante entonces determinar en la embarazada cuál es el riesgo de recurrencia de patología biliar y el asociado a realizar una intervención quirúrgica para poder tomar la decisión más adecuada. Según reportes de casos publicados, el riesgo de recurrencia de patología biliar tras un episodio de pancreatitis aguda durante el embarazo varía entre 6 a 50\% (Hernández et al., 2007; Eddy et al., 2008; Igbinosa et al., 2013) (Tabla 1), siendo mayor mientras más precoz ocurra el primer cuadro agudo durante el embarazo (Hernández et al., 2007; Eddy et al., 2008).

A pesar de que es ampliamente aceptado que el tratamiento de elección para la remoción de la vesícula en pacientes no embarazadas con colelitiasis es la colecistectomía laparoscópica, en los inicios de la era laparoscópica la gestación era considerada como contraindicación para la misma. Sin embargo, múltiples publicaciones en los últimos 15 años han demostrado que este procedimiento es seguro y eficaz en la paciente embarazada, siendo el momento ideal para su realización el segundo trimestre de gestación, con un riesgo de pérdida fetal del 0 - $5 \%$ y sin malformaciones atribuibles al procedimiento (Rollins et al, 2004; Date et al., 2008). Es más, se ha visto que las ventajas descritas para la laparoscopía son también aplicables a la mujer gestante, entre ellas cabe destacar: recuperación posoperatoria más rápida, estadías hospitalarias más cortas, menor dolor posoperatorio, realimentación más precoz, menos manipulación uterina y menor riesgo de trombosis venosa profunda gracias a la rápida recuperación de la movilidad en estas pacientes (Larraín et al., 2007; Date et al., 2008).

Existen en la literatura al menos 8 reportes de casos que incluyen 170 pacientes que fueron sometidas a colecistectomía durante el embarazo con excelentes resultados (Tabla 7) (Affleck et al., 1999; Rollins et al., 2004; Chiappetta et al., 2009). De este modo, la colecistectomía laparoscópica sería un procedimiento seguro en el embarazo; así, considerando el alto riesgo de recurrencia de patología biliar en la embarazada, con el riesgo materno y fetal subsecuente, la colecistectomía laparoscópica debe ser ofrecida a las embarazadas al momento del alta de un episodio de pancreatitis aguda.

Tabla 7: Colecistectomía laparoscópica en pacientes embarazadas.

\begin{tabular}{|c|c|c|c|c|c|}
\hline \multirow{2}{*}{ Referencia } & \multicolumn{3}{|c|}{ Trimestre de embarazo } & \multirow{2}{*}{$\begin{array}{l}\text { Total } \\
\text { pacientes }\end{array}$} & \multirow{2}{*}{ Comentarios } \\
\hline & I & II & III & & \\
\hline $\begin{array}{l}\text { Affeck et } \\
\text { al., } 1999\end{array}$ & 3 & 13 & 11 & 45 & $\begin{array}{l}5 \text { Partos } \\
\text { prematuros (PP) }\end{array}$ \\
\hline $\begin{array}{l}\text { Barone et } \\
\text { al., } 1999\end{array}$ & & & & 20 & $\begin{array}{l}1 \text { caso Síntoma } \\
\text { PP (SPP) }\end{array}$ \\
\hline $\begin{array}{l}\text { Cosenza et } \\
\text { al., } 1999\end{array}$ & 0 & 12 & 0 & 12 & $\begin{array}{l}2 \text { conversiones } \\
\text { laparotomía }\end{array}$ \\
\hline $\begin{array}{l}\text { Muench et } \\
\text { al., } 2001\end{array}$ & 3 & 11 & 2 & 16 & $\begin{array}{l}2 \text { conversiones } \\
\text { a laparotomía. } \\
4 \text { SPP todos } \\
\text { llegaron a } \\
\text { término }\end{array}$ \\
\hline $\begin{array}{l}\text { Patel \& } \\
\text { Veverka, } \\
2002\end{array}$ & 3 & 6 & 1 & 10 & $\begin{array}{l}2 \text { conversiones a } \\
\text { laparotomía }\end{array}$ \\
\hline $\begin{array}{l}\text { Rolling et } \\
\text { al., } 2004\end{array}$ & 3 & 19 & 9 & 31 & $\begin{array}{l}1 \text { Muerte fetal in } \\
\text { utero } 9 \text { semanas } \\
\text { posterior a } \\
\text { procedimiento. } \\
2 \text { partos } \\
\text { prematuros }\end{array}$ \\
\hline $\begin{array}{l}\text { Daradkeh, } \\
2005\end{array}$ & 4 & 11 & 5 & 20 & $\begin{array}{l}\text { Sin compli- } \\
\text { caciones }\end{array}$ \\
\hline $\begin{array}{l}\text { Chiappetta } \\
\text { et al., } 2008\end{array}$ & 0 & 54 & 0 & 54 & $\begin{array}{l}\text { Sin compli- } \\
\text { caciones }\end{array}$ \\
\hline $\begin{array}{l}\text { Fernández } \\
\text { et al., 2015* }\end{array}$ & 14 & 30 & 1 & 45 & $\begin{array}{l}\text { Sin compli- } \\
\text { caciones }\end{array}$ \\
\hline Total & 16 & 126 & 28 & 170 & \\
\hline
\end{tabular}

*Datos no publicada 
Otra herramienta en el manejo de pancreatitis aguda litiásica es la colangio-pancreatografía retrógrada endoscópica (CPRE), la cual tiene un rol diagnóstico y terapéutico, siendo sus indicaciones más aceptadas pancreatitis con coledocolitiasis asociada y la prevención de recurrencias en pancreatitis aguda litiásica durante el tercer trimestre de embarazo, lo cual permite una resolución quirúrgica definitiva en el postparto. Este procedimiento, al igual que la colecistectomía laparoscópica, ha demostrado ser seguro durante el embarazo. Una de las principales preocupaciones radica en la necesidad de radiación, la cual se estima en un rango de 0.1-3 mSv, lo cual se encuentra por debajo de la radiación permitida durante la gestación, la cual es de 5 mSv (Ducarme et al., 2014. Date et al. 2008). Por otra parte, algunos autores han publicado experiencia de CPRE exitosa sin uso de radiación durante el embarazo (Simmons et al., 2004; Shelton et al., 2008).

Proponemos siempre un manejo multidisciplinario en conjunto con el equipo de cirugía. En los casos del primer trimestre, diferir la cirugía hasta el segundo trimestre. En casos del segundo trimestre, realizar la resolución quirúrgica previo al alta y en los casos del tercer trimestre, evaluar caso a caso el mejor momento de resolución.

\section{Conclusiones}

A pesar de que la PA es una patología poco frecuente durante el embarazo, su espectro clínico es muy amplio, con cuadros leves de compromiso solo local, hasta compromiso sistémico importante e incluso muerte materna y /o fetal.

Su diagnóstico se basa en la presencia de dos de tres criterios diagnósticos: cuadro clínico, exámenes de laboratorio y/o estudio de imágenes.

Frente a un cuadro clínico sospechoso, se deben solicitar amilasa y/o lipasa, cuya elevación confirmará el diagnóstico. Dado que la etiología más frecuente es secundaria a patología biliar, se debe realizar siempre una ecografía abdominal para descartar colelitiasis asociada. El estudio con TAC se reserva para los casos de PA grave o deterioro clínico.

El manejo de la PA en la embarazada debe ser multidisciplinario, con énfasis en la reposición de fluidos, soporte nutricional precoz y analgesia adecuada. La antibioterapia se reserva para los casos de pancreatitis grave $\mathrm{y} / \mathrm{o}$ asociada a colangitis.

En cuanto a la resolución quirúrgica, existe controversia sobre las indicaciones y mejor momento de las intervenciones quirúrgicas. Proponemos en casos de PA secundarias a colelitiasis realizar colecistectomía; en los casos del primer trimestre, diferir la cirugía hasta el segundo trimestre. En casos del segundo trimestre, realizar la resolución quirúrgica previo al alta y en los casos del tercer trimestre, evaluar caso a caso el mejor momento de la colecistectomía.

\section{Referencias:}

Affleck, D. G., Handrahan, D. L., Egger, M. J., \& Price, R. R. (1999). The laparoscopic management of appendicitis and cholelithiasis during pregnancy. American Journal of Surgery, 178(6), 523-9.

Al-Omran, M., AlBalawi, Z. H., Tashkandi, M. F., \& Al-Ansary, L. A. (2010, January 20). Enteral versus parenteral nutrition for acute pancreatitis.

Almora Carbonell, C. L., Arteaga Prado, Y., Plaza González, T., Prieto Ferro, Y., \& Hernández Hernández, Z. (2012). Diagnóstico clínico y epidemiológico de la litiasis vesicular. Revisión bibliográfica. Revista de Ciencias Médicas de Pinar del Río 16. 1999 Evaluation. Radiology, 223(3), 603-613.

Balthazar, E. J., Robinson, D. L., Megibow, A. J., \& Ranson, J. H. (1990). Acute pancreatitis: value of CT in establishing prognosis. Radiology, 174(2), 331-336.

Barone JE, Bears S, Chen S, Tsai J \& Russell JC. (1999). Outcome study of cholecystectomy during pregnancy. Am J Surg. 177(3): 232-236.

Basurto Ona, X., Rigau Comas, D., \& Urrútia, G. (2013). Opioids for acute pancreatitis pain. In X. Basurto Ona (Ed.), Cochrane Database of Systematic Reviews (p. CD009179). Chichester, UK: John Wiley \& Sons, Ltd.

Burtin, P., Taddio, A., Ariburnu, O., Einarson, T. R., \& Koren, G. (1995). Safety of metronidazole in pregnancy: a meta-analysis. American Journal of Obstetrics and Gynecology, 172(2 Pt 1), 525-9.

Chiappetta Porras, L. T., Nápoli, E. D., Canullán, C. M., Quesada, B. M., Roff, H. E., Alvarez Rodríguez, J. (2009). Minimally invasive management of acute biliary tract disease during pregnancy. $H P B$ Surgery: A World Journal of Hepatic, Pancreatic and Biliary Surgery, 2009,829020 .

Cosenza CA, Saffari B, Jabbour N, Stain SC, Garry D, Parekh D, Selby RR. (1999). Surgical management of biliary gallstone disease during pregnancy. Am J Surg.178(6) 545-548.

Date, R. S., Kaushal, M., \& Ramesh, A. (2008). A review of the management of gallstone disease and its complications in pregnancy. The American Journal of Surgery, 196(4), 599-608.

Daradkeh S (2005). Laparoscopic cholecystectomy: analytical study of 1208 cases. Hepatogastroenterology.52 1011-1014 
Ducarme G, Maire F, Chatel P, Luton D \& Hammel P. (2014) Acute Pancreatitis during pregnancy: a review. Journal of Perinatology. 34 87-94.

Eddy, J. J., Gideonsen, M. D., Song, J. Y., Grobman, W. A., \& O'Halloran, P. (2008). Pancreatitis in pregnancy. Obstetrics and Gynecology, 112(5), 1075-81.

Gardner, T. B., Vege, S. S., Pearson, R. K., \& Chari, S. T. (2008). Fluid Resuscitation in Acute Pancreatitis. Clinical Gastroenterology and Hepatology, 6(10), 1070-1076.

Garel, C., Brisse, H., Sebag, G., Elmaleh, M., Oury, J.-F., \& Hassan, M. (1998). Magnetic resonance imaging of the fetus. Pediatric Radiology.28 201-211.

Hernández, A., Petrov, M. S., Brooks, D. C., Banks, P. A., Ashley, S. W., \&Tavakkolizadeh, A. (2007). Acute pancreatitis and pregnancy: A 10-year single center experience. Journal of Gastrointestinal Surgery, 11(12), 1623-1627.

Igbinosa, O., Poddar, S., \& Pitchumoni, C. (2013). Pregnancy associated pancreatitis revisited. Clinics and Research in Hepatology and Gastroenterology, 37(2), 177-181.

Karsenti, D., Bacq, Y., Brechot, J.-F., Mariotte, N., Vol, S., \& Tichet, J. (2001). Serum amylase and lipase activities in normal pregnancy: a prospective case-control study. The American Journal of Gastroenterology, 96(3), 697-699.

Koo, B. C., Chinogureyi, A., \& Shaw, A. S. (2010). Imaging acute pancreatitis. The British Journal of Radiology, 83(986), 104-12.

Larraín de la C, D., Durruty V, G., Pomés C, C., \& Cuello F, M. (2007). Consideranciones para el uso de la laparoscopía durante el embarazo. Revista Chilena de Obstetricia Y Ginecología, 72(4), 247-257.

Larsson, A., Palm, M., Hansson, L.-O., \& Axelsson, O. (2008). Reference values for clinical chemistry tests during normal pregnancy. $B J O G$ : An International Journal of Obstetrics \& Gynaecology, 115(7), 874-881.

Lazarus, E., Mayo-Smith, W. W., Mainiero, M. B., \& Spencer, P. K. (2007). $\mathrm{CT}$ in the Evaluation of Nontraumatic Abdominal Pain in Pregnant Women. Radiology, 244(3), 784-790.

Muench J, Albrink M, Serafini F, Rosemurgy A, Ca- rey L \& Murr MM (2001). Delay in treatment of biliary disease during pregnancy increases morbidity and can be avoided with safe laparoscopic cholecystectomy. Am Surg. 67:539-542.
Mutter, T. C., Ruth, C. A., \& Dart, A. B. (2013). Hydroxyethyl starch (HES) versus other fluid therapies: effects on kidney function. Cochrane Database Syst Rev., 23(7), CD007594.

Nuray Turhan, A., Gönenç, M., Kapan, S., İslim, F., Öner, O. Z., Tulubaş, E., ... Bölümü, R. (2010). Acute biliary pancreatitis related with pregnancy: a 5-year single center experience. Turkish Journal of Trauma \& Emergency Surgery, 16(151), 160-164.

Papadakis, E. P., Sarigianni, M., Mikhailidis, D. P., Mamopoulos, A., Karagiannis, V., \& Al-Manasra, A. R. (2011). Acute pancreatitis in pregnancy: an overview. European Journal of Obstetrics, Gynecology, and Reproductive Biology, 159(2), 261-6.

Patel SG \& Veverka TJ. (2002). Laparoscopic cholecystectomy in pregnancy. Curr Surg 59 74-78.

Pitchumoni, C. S., \& Yegneswaran, B. (2009). Acute pancreatitis in pregnancy. World Journal of Gastroenterology, 15(45), 5641-6.

Qihui, C., Xiping, Z., \& Xianfeng, D. (2012). Clinical study on acute pancreatitis in pregnancy in 26 cases. Gastroenterology Research and Practice, 2012, 271925. https://doi.org/10.1155/2012/271925

Ramin, K. D., Ramin, S. M., Richey, S. D., \& Cunningham, F. G. (1995). Acute pancreatitis in pregnancy. American Journal of Obstetrics and Gynecology, 173(1), 187-191.

Ramin, K. D., \& Ramsey, P. S. (2001). Disease of the gallbladder and pancreas in pregnancy. Obstetrics and Gynecology Clinics of North America, 28(3), 571-80.

Rinninella M.G, Anneta M.L, Serrichio A.A. Dal Lago G.A, Miggiano M.C, \& Mele M.C. (2017) Nutritional support in acute pancreatitis: from physiopathoogy to practice. European Review for Medical and Pharmacological Sciences. 21, 421-432.

Rollins, M. D., Chan, K. J., \& Price, R. R. (2004). Laparoscopy for appendicitis and cholelithiasis during pregnancy: a new standard of care. Surgical Endoscopy, 18(2), 237-241.

Russo-Stieglitz, K. E., Levine, A. B., Wagner, B. A., \& Armenti, V.T. (1999). Pregnancy outcome in patients requiring parenteral nutrition. The Journal of Maternal-Fetal Medicine, 8(4), 164-167.

Sarr, M. G. (2013). 2012 revision of the Atlanta classification of acute pancreatitis. Polskie Archiwum Medycyny Wewnętrznej, 123(3), 118-124. 
Scott, L. D. (1992). Gallstone disease and pancreatitis in pregnancy. Gastroenterology Clinics of North America, 21(4), 803-15.

Shelton J, Linder JD, Rivera-Alsina ME \&Tarnasky PR. (2008) Commitment, confirmation, and clearance: new techniques for nonradiation ERCP during pregnancy. Gastrointestinal Endoscopic. 67 364-368.

Simmons DC, Tarnasky RR, Rivera-Alsina ME, Lopez JF \& Edman CD. (2004). Endoscopic retrograde cholangiopancreatography (ERCP) in pregnancy without the use of radiation. American. Journal Obstetrics Gynecology. 190, 1467-1469.

Uribe, R., Sáez O, N., \& Carvajal C, J. (2009). Estudios de radiodiagnóstico durante el embarazo. Revista Chilena de Obstetricia $Y$ Ginecología, 74(2), 117-122.

Valdivieso, V., Covarrubias, C., Siegel, F., \& Cruz, F. (1993). Pregnancy and cholelithiasis: pathogenesis and natural course of gallstones diagnosed in early puerperium. Hepatology (Baltimore, Md.), 17(1), 1-4.

Vallano, A., \& Arnau, J. M. (2009). Antimicrobianos y embarazo. Enfermedades Infecciosas Y Microbiologia Clinica, 27, 536-542. van Baal, M. C., Besselink, M. G., Bakker, O. J., van Santvoort, H. C., Schaapherder, A. F., Nieuwenhuijs, V. B., ... Dutch Pancreatitis Study Group. (2012). Timing of cholecystectomy after mild biliary pancreatitis: a systematic review. Annals of Surgery, 255(5), 860-866.

Vonlaufen, A., Wilson, J. S., \& Apte, M. V. (2008). Molecular mechanisms of pancreatitis: Current opinion. Journal of Gastroenterology and Hepatology, 23(9), 1339-1348.

Working Group IAP/APA. (2013). IAP/APA evidence-based guidelines for the management of acute pancreatitis. Pancreatology. 13 e1-e15.

World Health Organization. (2011). Evaluating the quality of care for severe pregnancy complications The WHO near-miss approach for maternal health Evaluating the quality of care for severe pregnancy complications. The WHO near-miss approach for maternal health. www.who.int/reproductivehealth

Wu, B. U., Hwang, J. Q., Gardner, T. H., Repas, K., Delee, R., Yu, S., .. Conwell, D. L. (2011). Lactated Ringer's Solution Reduces Systemic Inflammation Compared With Saline in Patients With Acute Pancreatitis. Clinical Gastroenterology and Hepatology, 9(8), 710-717.

Yadav, D., \& Lowenfels, A. B. (2013). The epidemiology of pancreatitis and pancreatic cancer. Gastroenterology. 144 1252-1261 